\title{
NOTE ON THE NAME OF KING NARMER
}

The name of Narmer ( $\mathrm{n}$ ' $r-\mathrm{mr}$ ), king of Upper Egypt in the late predynastic period (ab. 3000 B. C.), has remained a mystery for long millennia. The first component of the name is clearly identical with n'r "Wels (catfish)" (OK, Med., Gr., Wb II 209, 2-6). But the second element $-\mathrm{mr}$ has so far been lacking a reliable and convincing etymological explanation on the Egyptian lexical material. In this brief paper we attempt to give a solution for the second component of the name in the Common Afrasian (Semito-Hamitic) lexical material. First, we can also admit that the Egyptian vocabulary does not help too much to clarify $-\mathrm{mr}$, as there is no Egyptian word to fit in the name.

1. One could expect -mr to mean among others "fish". In this case the whole name could have meant "Catfish". This kind of name of the rapacious Nile fish fits well with the names of the previous kings from the "0. Dynasty", probable ancestors of Narmer (predynastic period): k3 "Bull", srq "Scorpion" (cf. e. g. Kákosy 1993, 40). Just this kind of reasoning seems confirmed if the 2 nd component $-\mathrm{mr}$ phonologically reflects an earlier *ml, because this purely hypothetically reconstructed Eg. *mr "fish" would be a perfect match of the Common Afrasian noun *mwal- "fish", having its traces in ECu. Somali-Hawiya mallay, Somali-Digil malalay, Jäbärti of Somali mallây "fish" (Reinisch 1904, 78), Boni melelé'i "fish" (Heine 1977, 287), Sidamo mwoliyā "fish" (Cohen 1947, \#466) || | NOm. Chara mulā Wolamo mole, Zala muoliyā, Gofa molā, molo, Badditu malalā "fish", Gamu, Dache, Zayse, Zergulla mole, Dorze mollé, mole, Oyda mollo, molo, Dawro molya, Kullo mōliya. Malo, Kachama molo, Basketo mola, mola, Haruro molo, Gidicho mollo, Koyra malala || SOm. Ari mol-ta, Galila mola. Cf. Cerulli 1951, 172; Mukarowsky 1981, 208, \#32; Lamberti 1994, 115

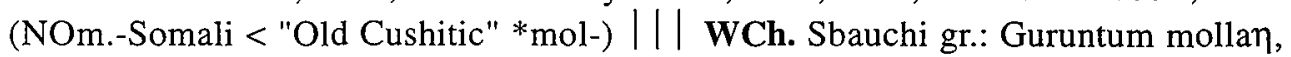
mwerrang "fish" (JI 1994, 140). It is not excluded that the ECu. forms represent a borrowing from various NOm. sources (Lamberti, pers. comm. on 6 January 1997).

Our hypothetically reconstructed Eg. word *mr, maybe, is not fully unattested within Egyptian, compare the obscure mr.t glossed by Erman and Grapow as "ein Tier (zwischen Fischen gennant)" (XVIII. Mag., Wb II 105, 17).

Note that Cohen (1947, \#466) has mistakenly equated the ECu. data with Eg. rm "fish" (OK, Wb II 416, 12). In theory, Eg. rm could represent a metathetical cognate (from *lam-) to the ECu.-SBauchi isogloss, but the Cop. reflexes (S) rame, raame, (B) rami "Nilbarsch" (Osing 1976, 429: < Eg. *rémjV[j/w] and Vycichl 1983, 172: 
"original" * rïmey, pl. *rimy-ū yielding later on *rēm, pl. *rame) make it clear that Eg. rm cannot be related. For the problem of true Afrasian cognates of Eg. rm "fish" see Takács 1995 and forthcoming.

2. The eventual further affiliation of the Omotic-East Cushitic-SBauchi (- ?Egyptian) isogloss *m[u]l- "fish" deserves a discussion here. Judging by some typological parallels (below, \#2.1), we cannot exclude that this form has preserved ultimately the same root as AA *mulh/"- "lizard", which is attested in Berb. Qabyle of Jurjura a-mulab "Algerian lizard (Tropidosaura algira)", Beni Menacer mulab "lizard" (Basset 1885, 174) || ECu. Sam gr. Rendille mulúh, Somali mula', mulu 'a "lizard" (Heine 1978, 69: PSam *mul'); Afar mullu'it "lizard" ||| WCh. Ron gr. Bokkos mulúsûs "grey lizard", cf. Hausa mulwa "short thick snake" || CCh. Kobochi malwā', Nzangi mālawá, Holma malwé "chameleon" (Strümpell 1922-1923, 135) || | ?Eg.: compare the second component -mnh in k3-mnh "Schildkröte" (Gr., Wb V 96, 9), k3being probably "turtle" from AA *kur- "turtle" (details in Takács 1996, \#53).

See Mukarowsky 1987, 245 (Bokkos-ECu.); Blažek 1992, 137 (AA *mul-: Ecu., WCh., CCh., Berb.); Orel-Stolbova 1992, 208 (ECu., WCh); Orel 1993, 39 (WCh., ECu.); HSED \#1792 (Bokkos-Hausa, ECu.).

The Berb. *-mulab should ba analyzed as *-mul-ab with the suffix *-b, which is attested in many Afrasian animal names, cf. Diakonoff 1965,28 , note 40,52 , note 2; 1967, 210; 1970, 461, note 23; 1975, 140; 1986, 47; 1988, 57; Fronzaroli 1969, 307, note 113, ; Illič-Svityč 1971, 192-193; Eilers 1978, 129; 1987, 516, 523.

For AA *mulh/"- "lizard" an areal parallel may be found in NMande *muluku "lizard" (Mukarowsky, loci cit.).

2.1. The eventual semantic dispersion between "lizard" "snake" "fish" is not unique in $\mathrm{AA}, \mathrm{cp}$. the following two Common Afrasian words:

2.1.1. AA * dV(n)g-ar, attested in: Wsem. Ug. dg "Fische" (coll.), dgy "Fischer", Hebr. dāg "Fische", dayyāg "Fischer" (WUS \#725) ||| ?Eg. dngngs "Bez. der Uraeusschlange" (Gr., Wb V 470, 8) || | LECu. Oromo dangal-ée "tadpole" ||| NOm. *dingar- "snake": Kaffa dingaro, dingiroo (Bender 1975, 187: díngérò), Gimirra dingar ||| WCh. Hausa k’a-dangara "lizard" (Kraft: ka-dangarèè) | Chip dł̀gos "lizard" | Bole-Tangale gr.: Karekare də̀nkarà, Ngamo dàkùra "lizard" || Cch.

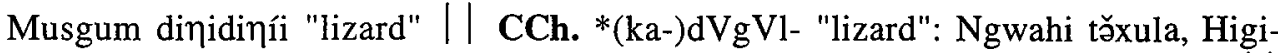
Nkafa xà-digàla, Fali-Kiriya (w)nžàxàla, Masa ho-donola, Banana hwǒ-dèngwălà ECh. Mubi danguda "Chamäleon" (Strümpell 1922-1923, 135).

See also Kraft 1981; Mukarovsky 1987, 244; Blažek 1989, 212; HSED \#716.

2.1.2. The Eg. word ‘š3 "lizard" (Pap. Ram. V, 21 [written ‘š]; Gardiner 1927, 465: I1; FD 49) is also most probably a reflex of $\mathbf{A A} *$ *a[ç]- "fish", preserved in Bed. aša, aše, Ammar'ar 'áašu "fish" || Agaw *'a[s]-: Bilin 'asa, Hamir xasa, Qemant asa, Awngi asi "fish" (Appleyard 1984, 44, 57; Agaw > Eth.-Sem.: Geez 'āsāa, 'aśa, Tigre, Tigrinya 'asa, Amhara asa "fish", Leslau 1988, 85) || LECu. Saho "āsa "fish" 
(borrowed < Agaw?) ||| NOm. Kafa hāš̄o, ǎšo "Fische (coll.)", cf. eš "pescare", Gofa áso "Fisch(e)" (Reinisch 1888, 265), Mocha, Anfillo hāšo, Bworo aso, Nao aša "fissh" ECh. Kera ačo "sardine" (HSED \#1085).

There have been recorded also some variant forms with voiced sibilants in Cushitic and Omotic: Agaw Bilin 'azā, Hamir xazā, Qemant, Qwara azā "fish" (Reinisch 1885, 33; 1887, 654) || SCu. Asa azā "fish" ||| NOm. Kaffa ážó, hāžó (Reinisch 1888, 265).

See Cohen 1947, \#48, Hintze 1951, 76; Dolgopol'skij 1973, 293; Bender 1975, 163; Appleyard 1977, 42/84; Zaborski 1989, 581; Orel-Stolbova 1992, 167.

\section{Abbreviations and special signs}

AA: Afroasiatic (Hamito-Semitic), Akk.: Akkadian, (B): Bohairic dialect of Coptic, BD: Book of the Dead, Bed.: Bed'awye, Berb.: Berber-Guanche, C: Central, Ch. Chadic, Cop.: Coptic, CT: Coffin Texts, Cu.: Cushitic, Drav.: Dravidian, E: East, Eg.: Egyptian, Eth.-Sem.: Ethio-Semitic, Gr.: Greek and Roman Period, H: Highland, Hebr.: Hebrew, IE: Indo-European, Kartv.: Kartvelian, L: Lowland, LP: Late Period, Med.: Mediacal Texts, Megr.: Megrelian, MK: Middle Kingdom, N: North, NK: New Kingdom, O: Old, OK: Old Kingdom, Om.: Omotic, P: Proto-, S: South, (S): Sahidic dialect of Coptic, Sem.: Semitic, Ug.: Ugaritic, W: West.

The vertical signs || $\mid$ indicate the closeness of relationship between the various Afrasian forms under discussion. Thus || separates two different branches (e. g. Cushitic and Chadic); || stands between sub-branches within the same branch (e. g. West Chadic and East Chadic); I marks the limit between groups within the same sub-branch (e. g. Kotoko group and Tera group in Central Chadic).

[ ] brackets indicate uncertain reconstruction, while ( ) mark a proto-phoneme omittable in the proto-form in question.

\section{References}

Appleyard, D. L.: A Comparative Approach to the Amharic Lexicin.= Afroasiatic Linguistics 5/2 (1977).

Appleyard, D. L.: The Internal Classification of the Agaw Languages. A Comparative and Historical Phonology.= Bynon, J. (ed.): Current Progress in Afro-Asiatic Linguistics. Amsterdam, 1984., John Benjamins. Pp. 33-67.

Basset, M. R.: Notes de lexicographie berbère.= Journal Asiatique. Ser. VIII, vol. 5 (1885), 148-198.

Bender, M. L.: Omotic. A New Afroasiatic language Familiy. Carbondale, Illinois, 1975., Southern Illinois University. 
Blažek, V.: A New Contribution to Comparative-Historical Afrasian Linguistics.= Asian and African Studies 24 (1989), 203-222.

Blažek, V.: Kartvelian Material in Nostratic Lexicon: New Ethymologies II.= Shevoroshkin, V. (ed.): Nostratic, Dene-Caucasian, Austric and Amerind. Bochum, 1992., Brockmeyer. Pp. 129-148.

Cerulli, E.: Studi etiopici. IV. La lingua caffina. Roma, 1951., Instituto per l'Oriente.

Cohen, M.: Essai comparatif sur le vocabulaire et la phonétique du chamito-sémitique.

Paris, 1947., Librairie Ancienne Honore Champion.

D'jakonov, I. M.: Semitohamitskie jazyki. Opyt klassifikacii. Moskva, 1965., Nauka.

D'jakonov, I. M.: jazyki Drevnej Perednej Azii. Moskva, 1967., Nauka.

Diakonoff, I. M.: On Root Structure in Proto-Semitic.= Bynon, J. and Bynon, Th.

(eds.): Hamito-Semitica. The Hague, 1975., Mouton. Pp. 133-153.

D'jakonov, I. M.: Obščeafrazijskie imennye kategorii.= Pis'mennye pamjatniki i problemy istorii kul'tury narodov Vostoka. XIX godičnaja naučnaja sessija LO IV AN SSSR. Moskva, 1986., Nauka. Pp. 47-62.

Diakonoff, I. M.: Afrasian Languages. Moscow, 1988., Nauka.

Dolgopol'sky, A. B.: Sravnitel'no-istoričeskaja fonetika kušitskih jazykov. Moskva, 1973., Nauka.

Eilers, W.: Semitische Wurzeltheorie.= Atti del Secondo Congresso Internazionale di Linguistica Camito-Semitica, Firenze, 16-19 aprile 1974. Firenze, 1978., Instituto Linguistica e di Lingue Orientali, Università di Firenze. Pp. 125-131.

Eilers, W.: Die zweiradikalige Basis der semitischen Wurzel.= Jungraithmayr, H.; Müller, W. W. (eds.): Proceedings of the Fourth International hamito-Semitic Congress, Marburg, 20-22 September, 1983. Amsterdam-Philadelphia, 1987., John Benjamins. Pp. 509-524.

$\mathrm{FD}=$ Faulkner, R. O.: A Concise Dictionary of Middle Egyptian. Oxford, 1980., Clarendon press.

Fronzaroli, P.: Studi sul Lessico Comune Semitico. VI.= Rendiconti delle Sedute dell'Accademia nazionale dei Lincei. Classe di scienze morali, storiche e filologiche. Ser. VIII, vol. XXIV, fasc. 7-12 (1969), 285-320.

Gardiner, A. H.: Egyptian Grammar. ${ }^{1}$ Oxford, 1927., Clarendon Press.

Heine, B.: Bemerkungen zur Boni-Sprache (Kenia).= Afrika und Übersee 60 (1977), 242-295.

Heine, B.: The Sam Languages. A History of Rendille, Boni and Somali.= Afroasiatic Linguistics 6/2 (1978), 23-115.

Hintze, Fr.: Zur hamitosemitischen Wortvergleichung.= Zeitschrift für Phonetik und Allgemiene Sprachwissenschaft 5 (1951), 65-87.

HSED = Orel, V. É.; Stolbova, O. V.: Hamito-Semitic Etymological Dictionary. Leiden, 1995., E. J. Brill.

Illič-Svityč, V. M.: Opyt sravnenija nostratičeskih jazykov. Vvedenie. Sravnitel'nyj slovar' (b-K'). Moskva, 1971. Nauka.

JI = Jungraithmayr, H. and Ibriszimow, D.: Chadic Lexical Roots. Vol. II. Documentation. Berlin, 1994., Dietrich Reimer Verlag.

Kákosy, L: Ré fiai. ${ }^{2}$ Budapest, 1993., Gondolat.

Kraft, Ch. H.: Chadic Worldlists. Vol. I-III. berlin, 1981., Dietrich Reimer Verlag. 
Lamberti, M.: Sulla classificazione dell'"Omotico".= Brugnatelli, V. (ed.): Sem, Cam, Iafet. Atti della $7^{\underline{a}}$ Giornata di Studi Camito-Semitici e Indoeuropei (Milano, 1ํo giugnio 1993). Milano, 1994., Centro di Studi Camito-Semitici. Pp. 99-126.

Leslau, W.: Analysis of the Ge'ez Vocabulary: Ge'ez and Cushitic.= Rassegna di Studi Etiopici 32 (1988), 59-109.

Mukarowsky, H. G.: Wo steht das Saharische?= Afrika und Übersee 64 (1981), 187226.

Mukarowsky, H. G.: Mande-Chadic Common Stock. Wien, 1987., Afro-Pub.

Newman, P. and Ma, R.: Comparative Chadic: Phonology and Lexicon.= Journal of African Languages 5/3 (1966), 218-251.

Orel, V. É. and Stolbova, O. V.: Cushitic, Chadic and Egyptian: Lexical Relations.= Shevoroshkin, V. (ed.): Nostratic, Dene-Caucasian, Austric and Armenid. Bochum, 1992., Brockmeyer. Pp. 167-180.

Orel, V. É.; Stolbova, O. V.: Position of the Cushitic (Preliminary Report).= Shevoroshkin, V. (ed.): Nostratic, Dene-Caucasian, Austric and Armenid. Bochum, 1992., Brockmeyer. Pp. 204-224.

Orel, V. É.: Mir semito-hamitov. Tezisy dokladov konferencii 8-9 nojabrja $1993 \mathrm{~g}$. Moskva, 1993., Institut Jazykoznanija Rossijskoj Akademii Nauk. Pp. 37-44.

Orel, V. É.: Reconstructing the Homeland of Proto-Afroasiatic. MS. Paper read at the 3rd World Archaeological Congress, New Delhi, December 1994a. 8 p.

Osing, J.: Die Nominalbildung des Ägyptischen. I-II. Mainz/Rhein, 1976., Philipp von Zabern.

Reinisch, L.: Die Quarasprache in Abessinien. II.= Sitzungsberichte der Kaisserlichen Akademie der Wissenschaften. Phil.-hist. Classe 109/1 (1885), 3-152.

Reinisch, L.: Die Quarasprache in Abessinien. III.= Sitzungsberichte der Kaisserlichen Akademie der Wissenschaften. Phil.-hist. Classe 114 (1887), 639-688.

Reinisch, L.: Die Kafa-Sprache in Nordost-Afrika. II.= Sitzungsberichte der Kaisserlichen Akademie der Wissenschaften. Phil.-hist. Classe 116 (1888), 251-386.

Reinisch, L.: Der Dschäbärtidialekt der Somalisprache.= Sitzungsberichte der Kaisserlichen Akademie der Wissenschaften. Phil.-hist. Classe 148/5 (1904), 1-88

Strümpell, F.: Wörterverzeichnis der Heidensprachen des Mandara-Gebirges (Adamaua). Schluss.= Zeitschrift für Eingeborenen-Sprachen 13 (1922-23), 109_ 149.

Takács, G.: The Afrasian Origin of Egyptian rm "Fish" (Preliminary Version).= Koval', A. I.; Vinogradov, V. A. (reds.): Problemy izučenija jazykov Afriki. Materialy konferencii posvjačennoj 30-letiju Otdela afrikanskih jazykov Instituta Jazykoznanija Rossijskoj Akademii Nauk (Moskva, 4-6 dekabrja 1995 g.). Moskva, 1995., Institut Jazykoznanija RAN. Pp. 159-164.

Takács, G.: The Afrasian Origin of Egyptian rm "Fish".= Piekarski, Pr. (ed.): Papers of the Memorial Session in Honour of Preofessor Roman Stopa, Cracow, 9 December 1995. Cracow, forthcoming, Institute of Oriental Philology of the Jagello University.

Takács, G.: Egyptian Lexics in an Afrasian Perspective: New Etymologies.= Studia Etymologica Cracoviensia 1 (1996). In press.

Vycichl, W.: Dictionnaire étymologique de la langue copte. Leuven 1983., Peeters. 
$\mathrm{Wb}=$ Erman A. and Grapow H.: Wörterbuch der ägyptischen Sprache. I-V. ${ }^{2}$ Berlin, 1957-1971., Akademie Verlag.

WUS = Aistleitner, J.: Wörterbuch der ugaritischen Sprache.= Berichte über die Verhandlungen der Sächsischen Akademie der Wissenschaften zu Leipzig. Phil.-hist. Klasse 106/3 (1963).

Zaborski, A.: Der Wortschatz der Bedscha-Sprache - eine vergleichende Analyse.= Schuler, E. von (ed.); XXIII. Deutscher Orientalistentag, vom 16. bis 20. September 1985 in Würzburg. Ausgewählte Vorträge. Stuttgart, 1989., Franz Steiner Verlag. Pp. 573-591.

Povzetek

O IMENU KRALJA NARMERJA

Pisec vidi v imenu egiptovskega kralja Narmerja zloženko, katere prva sestavina je že prepoznana kot n'r 'som'. V drugi sestavini mr prepoznava neko slovarsko enoto s pomenom 'riba', ki je v egiptovčini slabo izpričana, zato pa toliko bolj v semio-hamitskem in drugem afrazijsem besednem zakladu. 